\title{
Frontal dermoid cyst coexisting with suprasellar craniopharyngioma: a spectrum of ectodermally derived epithelial-lined cystic lesions?
}

\author{
Hussam Abou-Al-Shaar, MD, ${ }^{1,2}$ Muhammad M. Abd-El-Barr, MD, PhD, ${ }^{2}$ Hasan A. Zaidi, MD, ${ }^{2}$ \\ Eleanor Russell-Goldman, MD, PhD, ${ }^{3}$ Rebecca D. Folkerth, MD, ${ }^{3}$ Edward R. Laws Jr., MD, ${ }^{2}$ and \\ E. Antonio Chiocca, MD, PhD² \\ ${ }^{1}$ College of Medicine, Alfaisal University, Riyadh, Saudi Arabia; and Departments of ${ }^{2}$ Neurosurgery and ${ }^{3}$ Pathology, Brigham and \\ Women's Hospital, Harvard Medical School, Boston, Massachusetts
}

\begin{abstract}
There is a wide group of lesions that may exist in the sellar and suprasellar regions. Embryologically, there is varying evidence that many of these entities may in fact represent a continuum of pathology deriving from a common ectodermal origin. The authors report a case of a concomitant suprasellar craniopharyngioma invading the third ventricle with a concurrent frontal lobe cystic dermoid tumor. A 21 -year-old man presented to the authors' service with a 3-day history of worsening headache, nausea, vomiting, and blurry vision. Magnetic resonance imaging depicted a right frontal lobe lesion associated with a separate suprasellar cystic lesion invading the third ventricle. The patient underwent a right pterional craniotomy for resection of both lesions. Gross-total resection of the right frontal lesion was achieved, and subtotal resection of the suprasellar lesion was accomplished with some residual tumor adherent to the walls of the third ventricle. Histopathological examination of the resected right frontal lesion documented a diagnosis of dermoid cyst and, for the suprasellar lesion, a diagnosis of adamantinomatous craniopharyngioma. The occurrence of craniopharyngioma with dermoid cyst has not been reported in the literature before. Such an association might indeed suggest the previously reported hypothesis that these lesions represent a spectrum of ectodermally derived epithelial-lined cystic lesions. https://thejns.org/doi/abs/10.3171/2016.8.FOCUS16238
\end{abstract}

KEY WORDS craniopharyngioma; third ventricle; intraaxial dermoid cyst; cystic intracranial lesions; embryology

$\mathrm{T}$ HERE is a wide spectrum of pathology that may exist in the sellar and suprasellar regions..$^{32}$ These entities include a spectrum of nonneoplastic lesions including Rathke's cleft cysts (RCCs), craniopharyngiomas, and epidermoid and dermoid cysts. There is varying evidence that many of these pathologies may in fact represent a continuum of pathology deriving from a common ectodermal origin. ${ }^{11}$ Herein, to our knowledge we report the first case of a concomitant suprasellar craniopharyngioma invading the third ventricle and a frontal lobe dermoid cyst in a 21-year-old man.

\section{Case Report}

History and Examination

A 21-year-old otherwise healthy man presented to our service with a 3-day history of worsening headache, nau- sea, and vomiting. The patient noted that over the past year he was having occipital headaches with associated auras in the form of blurry vision, which resolved following the start of medical management with clonazepam. Over the period of 3 days, however, he started having severe fluctuating headaches that were worse in the morning and associated with blurry vision and numbness of his extremities, as well as nausea and vomiting. The patient reported an episode of bowel incontinence and 2 episodes of unsteady gait and slurred speech over the previous 2 days. He had no family history of malignancy or neurological diseases.

On examination, the patient's vital signs were normal. $\mathrm{He}$ was alert and oriented to time, place, and person. He was neurologically intact, with normal visual fields to confrontation; the remainder of his examination was unremarkable. 

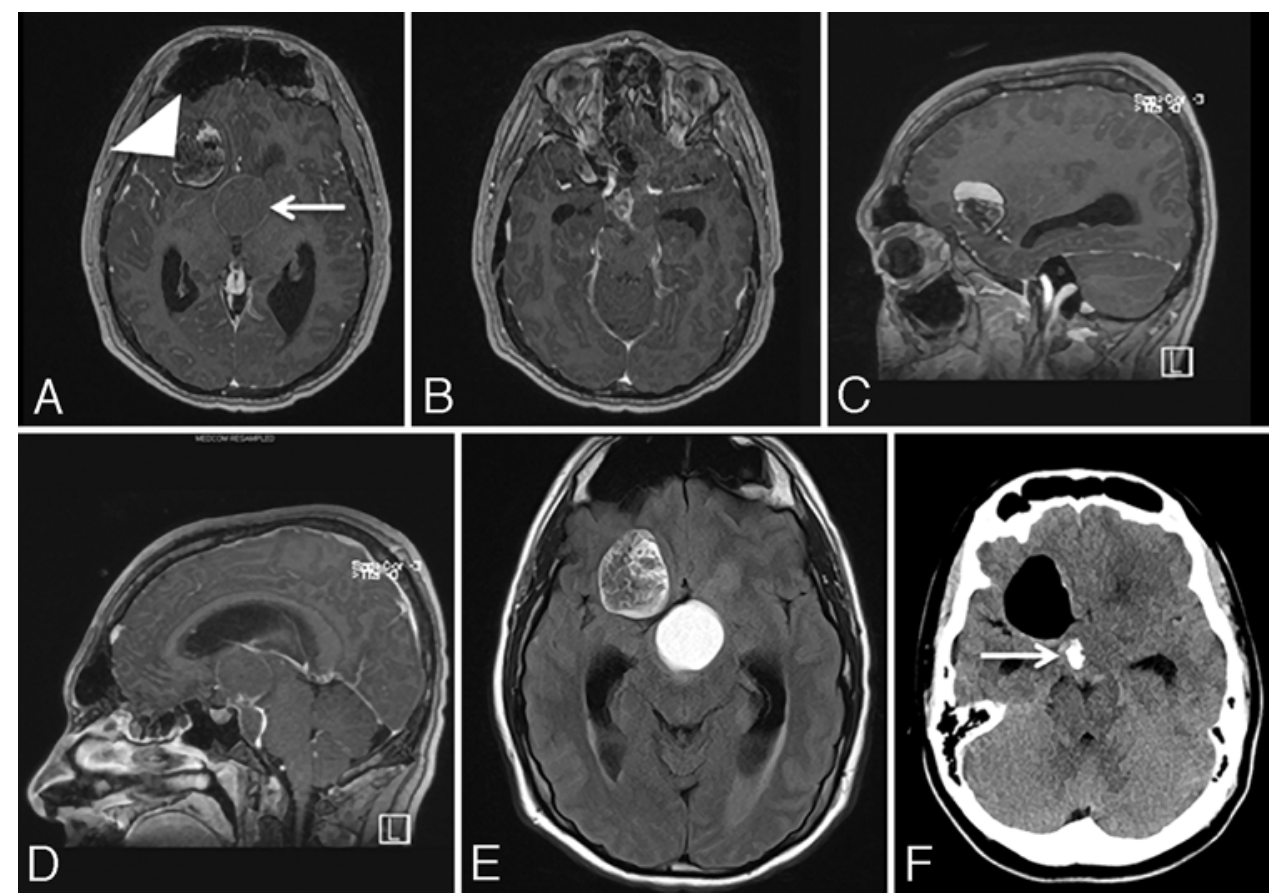

FIG. 1. Preoperative images. A: Axial T1-weighted contrast-enhanced MR image revealing a large, primarily suprasellar lesion (arrow). There is also a large right frontal lobe lesion with heterogeneous enhancement (arrowhead). B: Moving more caudally, the suprasellar lesion appears to have a smaller inferior component with a small blood/fluid level that extends into the prepontine cistern with posterior displacement of the basilar artery. C: Sagittal T1-weighted contrast-enhanced MR image showing that the right frontal lesion had heterogeneous enhancement. D: The suprasellar lesion appears to have 2 components-a larger cystic component and a smaller inferior component that extends into the prepontine cistern. E: Axial FLAIR MR image revealing that the suprasellar cyst is bright and homogeneous, while the right frontal lesion is heterogeneous. F: Axial CT scan revealing the right frontal lesion and the suprasellar lesion. The contents of the right frontal lesion are most likely fat, as the Hounsfield units are intermediate to those of air and CSF. There is also evidence of calcification in the suprasellar lesion (arrow).

\section{Imaging Studies and Laboratory Evaluation}

Magnetic resonance imaging depicted a $2.7 \times 3.4-\mathrm{cm}$ cystic T2 hyperintense lesion in the right frontal lobe associated with a $3.1 \times 2.9 \times 3.1-\mathrm{cm}$ primarily suprasellar cystic lesion with a proteinaceous component invading the third ventricle. The suprasellar lesion had a large superior and a smaller inferior component with a small blood/fluid level that extended into the prepontine cistern, with posterior displacement of the basilar artery, causing significant acute hydrocephalus. At the junction of the larger superior and smaller inferior cystic component there was a small solid, heterogeneous enhancing area slightly to the right of the midline (Fig. 1). It was clear that the frontal lesion and suprasellar lesion were distinct and not connected. The patient had significant hydrocephalus, sparing the fourth ventricle.

The hormonal laboratory tests demonstrated elevated prolactin $(72 \mathrm{ng} / \mathrm{ml})$ (reference range $2-18 \mathrm{ng} / \mathrm{ml}$ ) and insulin-like growth factor-1 $(474 \mathrm{ng} / \mathrm{ml})$ (reference range $115-345 \mathrm{ng} / \mathrm{ml}$ ). His follicle-stimulating hormone level was slightly low at $0.9 \mathrm{mIU} / \mathrm{ml}$ (reference range $1.5-12.4$ $\mathrm{mIU} / \mathrm{ml})$. His luteinizing hormone level was slightly low at $1.4 \mathrm{IU} / \mathrm{L}$ (normal 1.8-8.6 IU/L). His remaining laboratory results were within normal limits.

\section{Hospital Course and Index Operation}

The patient was admitted to the neurosurgical service, and an emergent external ventricular drain (EVD) was placed. The opening pressure was greater than $30 \mathrm{~cm} \mathrm{H}_{2} \mathrm{O}$. The patient reported immediate relief of his headaches after EVD insertion.

The next day, in the operating room, under neuronavigation, a right pterional craniotomy was planned in order to approach both lesions. The patient was placed supine with his neck extended and head turned at a $45^{\circ}$ angle toward the left. A right pterional incision was made, and a generous craniotomy flap was fashioned after a pericranial flap was harvested. The frontal sinus was intentionally opened, and the posterior wall of the frontal sinus and its mucosa were removed. Cerebrospinal fluid was aspirated to relax the brain for gentle frontal lobe retraction. The olfactory tract was identified, and a small corticectomy in the subfrontal area was established. Using the operating microscope and microdissection, the frontal lesion was encountered and was noted to be filled with hair, as well as a sebaceous-like substance (Fig. 2A and B). With meticulous microdissection, the cyst was removed entirely with no spillage of its contents into the surrounding brain. The frontal lobe was retracted further, and the right optic nerve was identified (Fig. 2C). The arachnoid plane between the optic nerve and the stalk area was opened, and the optic chiasm, the contralateral optic nerve, and the $A_{1}$ and $\mathrm{A}_{2}$ segments of both anterior cerebral arteries were visualized (Fig. 2D). The lamina terminalis was protrud- 

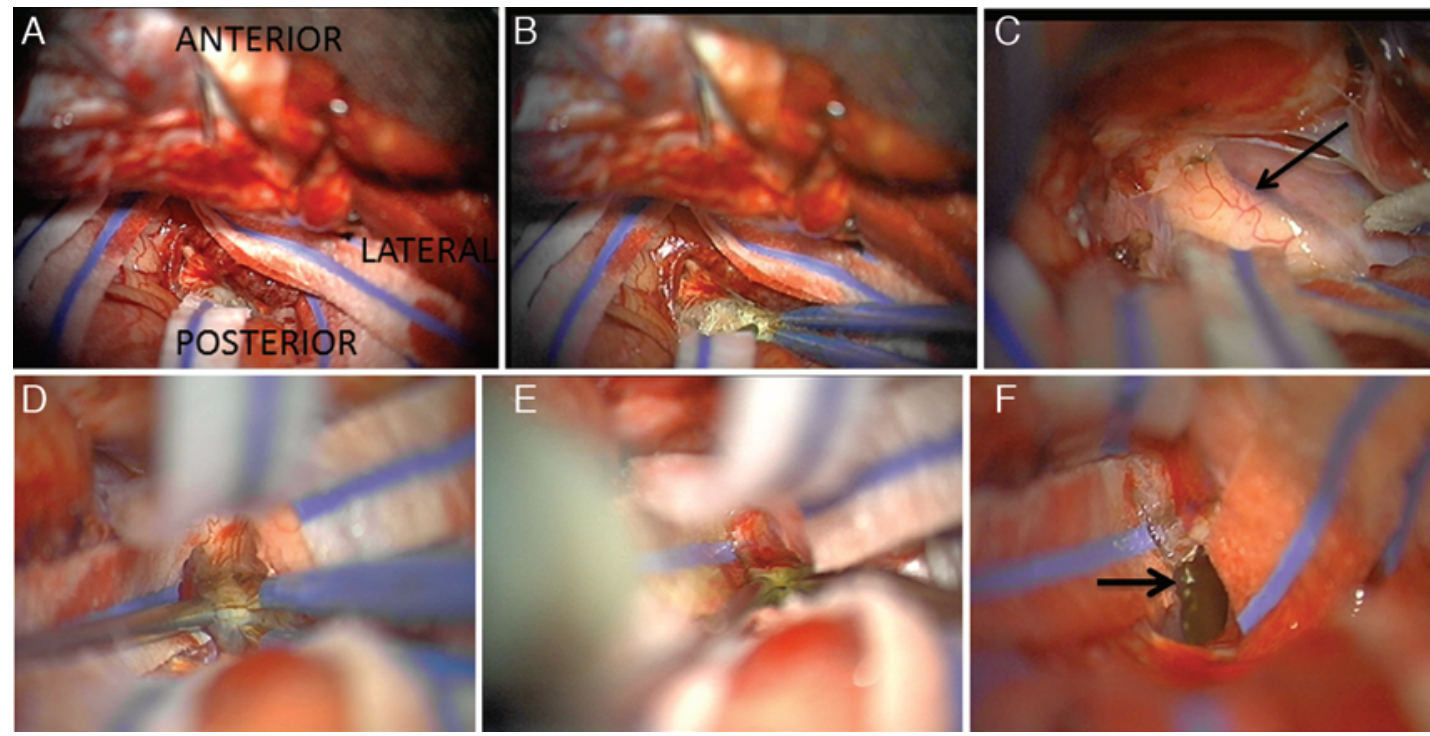

FIG. 2. Intraoperative images from the pterional approach. A: The subfrontal approach allowed for egress of CSF and minimal frontal lobe retraction. B: The frontal lesion was encountered after a small corticectomy, as shown by the blue bipolar prongs. This lesion was filled with hair, as well as a sebaceous-like substance. C: After careful evacuation of the frontal lesion, the frontal lobe was retracted further and the right optic nerve was identified (arrow). D: The arachnoid plane between the optic nerve and the stalk area was opened, and the optic chiasm, the contralateral optic nerve, and the $A_{1}$ and $A_{2}$ segments of both anterior cerebral arteries were visualized. E: The lamina terminalis was protruding, and the area was widely opened to identify the tumor. The tumor was visualized, and greenish oil-like fluid was oozing from it. F: In certain areas, the tumor appeared to be more attached to its surroundings than in others, and areas of calcification were clearly seen (arrow).

ing, and this area was widely opened to expose the tumor. The tumor was visualized and it contained greenish oil-like fluid (Fig. 2E). The tumor was decompressed and dissected from the walls of the third ventricle. In certain areas, the tumor appeared to be more adherent to its surroundings than in others, and areas of calcification were clearly seen (Fig. 2F). Inferiorly, the tumor extended into the prepontine area, and it also was attached to the pituitary stalk. The parts of the tumor that were attached too tightly to the walls of the third ventricle, to the stalk or inferiorly to the prepontine cistern, were not completely removed. The dura mater was closed, and the pericranial flap was placed over the frontal sinus and sutured to the dura in the subfrontal area. No intraoperative complications were encountered. The patient was safely extubated and transferred to the neurosurgical intensive care unit in good condition.

\section{Histopathological Examination}

Histopathological examination of the resected right frontal lesion showed typical histological components of a dermoid cyst, including numerous sebaceous glands and hair follicles (Fig. 3A). Immunohistochemical staining demonstrated a membranous and cytoplasmic betacatenin staining pattern in the dermoid cyst components, with absence of nuclear staining (Fig. 3B). In contrast, histological sections of the suprasellar lesion showed classic features of an adamantinomatous craniopharyngioma, including basal epithelium and squamous ghost cells referred to as "wet" keratin, with focal giant cell reaction (Fig. 3C). Immunohistochemical staining demonstrated the presence of nuclear beta-catenin in the supra- sellar lesion, again consistent with a craniopharyngioma (Fig. 3D).

\section{Postoperative Course and Follow-Up}

The patient recovered well and was treated with a diabetes insipidus protocol. He was neurologically stable. Postoperative MRI scans demonstrated gross-total resection of the right frontal lesion, with a very small focus in the right frontal resection bed likely representing small residual fragments (Fig. 4). Additionally, there was a residual of the suprasellar lesion that was adherent to the walls of the third ventricle (Fig. 4). The patient tolerated a weaning trial of the EVD, with stable ventricular size on repeat CT scans. The EVD was removed on postoperative Day 7 and the patient was discharged home on postoperative Day 8 in good condition.

After the index procedure, the patient was monitored with serial imaging. Approximately 2 months postoperatively, there was evidence of progressive enlargement of both the solid suprasellar component as well as a large cystic component extending into the interpeduncular space with compression of the brainstem (Fig. 5A and B). The patient had no discernable clinical deficits related to mass effect. After discussing treatment options, including observation, the patient elected for resection of the large cystic prepontine component of the mass followed by focused proton beam radiation therapy to the solid component. A direct approach using an endonasal endoscopic transclival exposure to remove this lesion was felt to provide the best access to the lesion. After performing a wide sphenoidectomy with removal of the vomer, the clivus was noted to be remodeled by the cystic posterior fossa mass. Exposure 


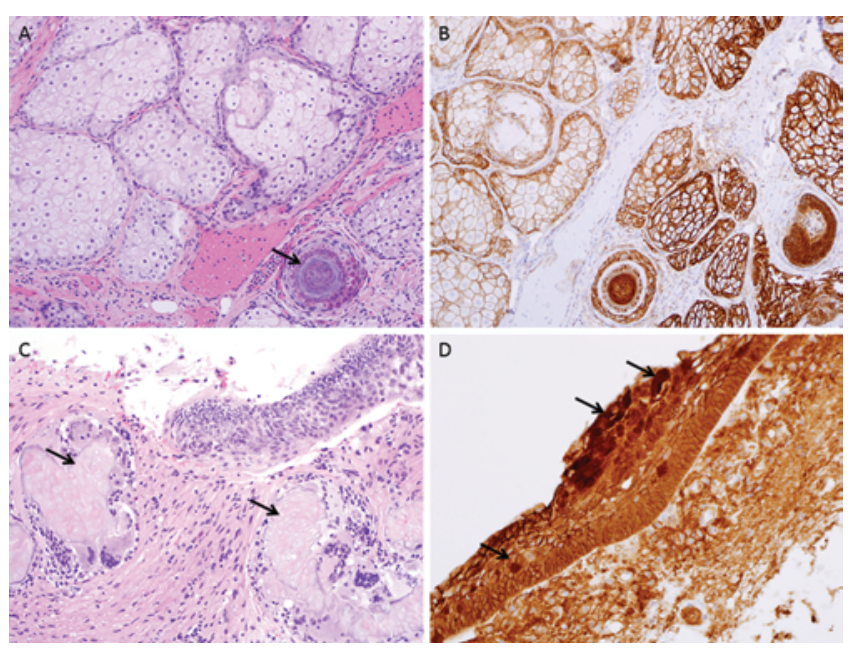

FIG. 3. Histological examination of the resected specimens. A: H \& Estained section of the frontal lesion showing typical histological components of a dermoid cyst, including numerous sebaceous glands and hair follicles (arrow). B: Immunohistochemical staining demonstrating membranous and cytoplasmic beta-catenin staining pattern in the dermoid cyst components with absence of nuclear staining. C: $\mathrm{H}$ \& E-stained section of the suprasellar lesion, showing classic histological features of an adamantinomatous craniopharyngioma, including basaloid epithelium and squamoid ghost cells referred to as "wet" keratin (arrows), with focal giant cell reaction. D: Immunohistochemical staining demonstrating the presence of nuclear beta-catenin (arrows) in the craniopharyngioma. Original magnification $\times 200(A-C)$ and $\times 400$ (D)

of the posterior fossa dura was obtained using drilling and rongeurs, and a circular dural opening was performed (Fig. 5C and D). The cystic lesion was decompressed, and the capsule was removed using pituitary graspers and counter-traction, with no evidence of residual disease on intraoperative inspection using angled endoscopes (Fig. $5 \mathrm{E}$ and F). A combination of fat graft, allograft dural substitute, and a Medpore plate (Porex) was used in a "gasket-seal" fashion to reconstruct the skull base defect. Postoperatively, the patient experienced transient diabetes insipidus, but otherwise had no new neurological deficits. Postoperative imaging demonstrated excellent resection of the posterior fossa cystic component of the tumor as well as a small amount of the solid component (Fig. 5G and $\mathrm{H}$ ), and pathology confirmed a residual adamantinomatous craniopharyngioma, identical to the previous pathology with regard to histological appearance. The patient was discharged home on postoperative Day 3, and he received focal proton beam radiation therapy to the solid parasellar component of the residual lesion.

\section{Discussion}

Various sellar and suprasellar cystic lesions demonstrate overlapping imaging and histological features, making their diagnosis extremely challenging. ${ }^{11}$ These lesions include craniopharyngiomas, RCCs, arachnoid cysts, and epidermoid and dermoid cysts. Harrison et al. reviewed 19 such cases and found great overlap in their clinical presentations, biological behaviors, and radiographic and histological features. ${ }^{11}$ The authors suggested that this overlap might be attributed to the varying degrees of dif-
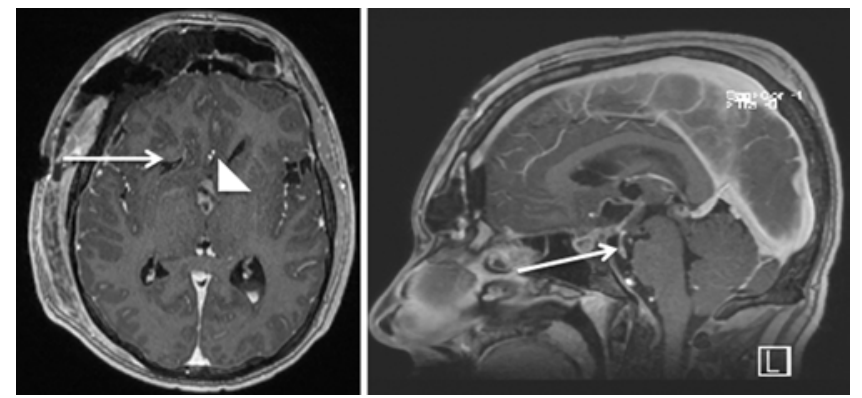

FIG. 4. Immediate postoperative MR images obtained after the index procedure. Left: Axial contrast-enhanced T1-weighted image showing gross-total resection of right frontal dermoid cyst (arrow). There is a residual of the suprasellar lesion that was adherent to the walls of the third ventricle (arrowhead). Right: Sagittal image showing a dramatic decrease in the size of the suprasellar lesion, although there is still some tumor in the interpeduncular cistern (arrow).

ferentiation, with the least differentiated lesions demonstrating overlapping features and the well-differentiated ones showing distinct characteristics. Therefore, based on these observations, the authors concluded that these epithelial-lined cystic lesions might share a common ectodermal progenitor and could be considered to be along a continuous spectrum. It has been our experience that there does indeed appear to be a wide spectrum in the histopathological differentiation of these suprasellar lesions, with some lesions being more differentiated than others. ${ }^{16}$ Another difficulty in the diagnosis is the inflammatory reaction that occurs in the adjacent pituitary gland and the various contents of the associated cysts. ${ }^{16}$

In conjunction with this hypothesis, in vitro studies of rats that underwent surgical implantation of epidermis and oral mucosa into their brains showed that these rats developed both epidermoid and dermoid cysts, suggesting a common ancestral origin. ${ }^{30,31}$ Interestingly, Kurwale et al. reported a case of a suprasellar dermoid cyst associated with a colloid cyst of the third ventricle in a 22-year-old man. ${ }^{14}$ Similarly, Jallo and colleagues reported a case of a colloid cyst associated with frontal epidermoid cyst in a 15 -year-old male patient, ${ }^{13}$ and Cheng et al. reported the simultaneous occurrence of a third ventricular colloid cyst and a nasal dermoid cyst in a 16-year-old male patient. ${ }^{5}$ Alomari et al. recently described a 7-year-old child with a sellar lesion, which on histopathological examination revealed areas of simple RCC morphology and areas of typical craniopharyngioma morphology. ${ }^{1}$ These observations and others have led many authors to believe that these cystic lesions are the result of disordered embryogenesis, thereby supporting the ectodermal progenitor theory for these cystic lesions.

From an embryological perspective, craniopharyngiomas are thought to arise from remnants of Rathke's pouch or squamous metaplasia within the pars tuberalis of the pituitary gland, while dermoid cysts are thought to arise from sequestration of totipotential cutaneous ectodermal cells into the developing neural tube during primary neurulation. ${ }^{6,15,18,19,23}$ Normally, the pituitary gland is formed by fusion of the ectoderm of the oral cavity stomodeum with the neuroectoderm of the diencephalon during the 3rd and 4th weeks of gestation. This fusion of the stomode- 

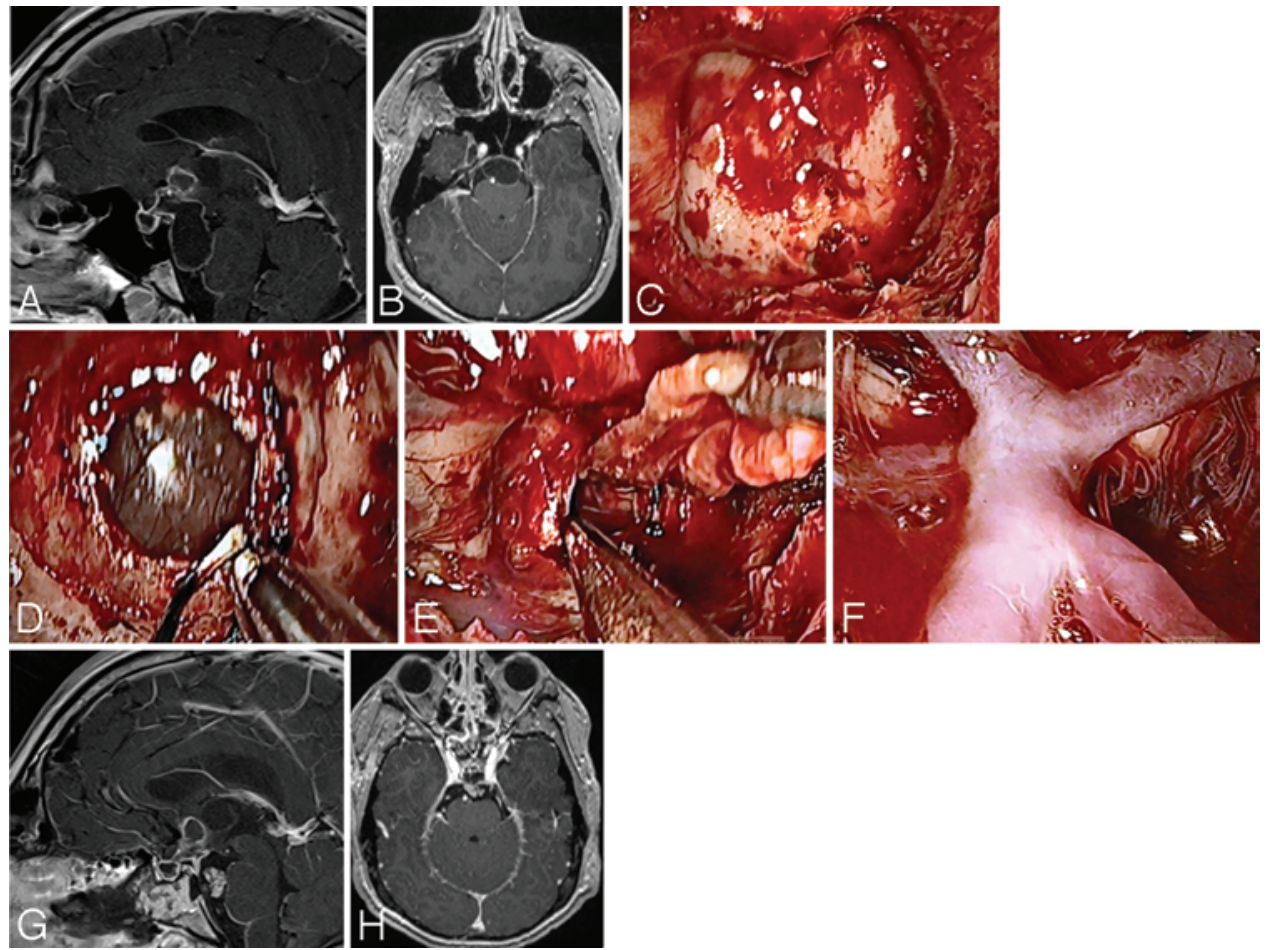

FIG. 5. Preoperative (second, transsphenoidal operation) Gd-enhanced sagittal (A) and axial (B) MR images demonstrating a heterogeneously enhancing, partially cystic mass in the suprasellar region with a large cystic component extending inferiorly to the interpeduncular cisterns, causing mass effect on the midbrain and pons. An endoscopic endonasal transclival approach was taken to resect the cystic component. C: A wide clival exposure was made by resecting the vomer and performing a wide sphenoidotomy. D: The cystic component had remodeled the clivus, which was removed using a Kerrison punch. E: The cystic lesion was decompressed, and $30^{\circ}$ angled endoscopes were used to inspect the cavity for residual disease. F: There was no evidence of residual tumor, with an excellent view of the basilar artery bifurcation, brainstem, and mammillary bodies. $\mathbf{G}$ and $\mathrm{H}$ : Postoperative Gd-enhanced sagittal $(\mathrm{G})$ and axial $(\mathrm{H})$ MR images demonstrating excellent decompression and resection of the cystic segment of the lesion and the position of the T1-hyperintense fat graft used to successfully repair the ventral skull base defect.

um and neuroectoderm gives rise to the adenohypophysis and neurohypophysis, respectively. ${ }^{2,12}$ During the process, a cleft forms between the anterior and posterior lobes of the hypophysis, giving rise to Rathke's cleft. It is therefore postulated that remnants of the stomodeum (i.e., Rathke's cleft and pharyngohypophyseal stalk) are the embryological progenitors of ectodermally derived epithelial-lined cystic lesions in the sellar and suprasellar regions, including RCCs, craniopharyngiomas, and dermoid cysts. ${ }^{25,26}$

The current case presents novel findings that were not previously reported, namely, the occurrence of a suprasellar craniopharyngioma with a dermoid cyst. The finding of nuclear staining for beta-catenin has been shown to be specific to adamantinomatous craniopharyngiomas, 3,17 which was found during histopathological study of the suprasellar lesion. This finding is thought to be due to overactivation of the WNT pathway, which has been found to be central to the pathophysiology of this subtype of craniopharyngioma. ${ }^{17}$ Moreover, the location and pattern of growth of these lesions are quite unusual. Craniopharyngiomas are suprasellar lesions that frequently invaginate the floor of the third ventricle..$^{27,28}$ Their extension vertically to invade the third ventricle is not common. Pascual et al. referred to such lesions as secondary third ventricular craniopharyngiomas. In addition, dermoid cysts tend to occur along the midline. ${ }^{21,22}$ They are most commonly encountered in the posterior fossa cisterns, parasellar cisterns, and fourth ventricle. ${ }^{10}$ Their occurrence inside the brain parenchyma is not commonly encountered..$^{8,9,24,29} \mathrm{In}$ a recent review of all intraaxial dermoid cysts, the authors found only 4 cases of frontal dermoid cysts, emphasizing the novelty of our case. . $^{8,20,29}$

Since gross-total resection is the mainstay of treatment for both of these lesions, ${ }^{4,7}$ the patient in our case underwent resection of both lesions via the pterional approach. The pterional approach was favored as it allowed optimal operative visualization of both lesions. In addition, opening the lamina terminalis was essential to visualizing the anterior part of the third ventricle and the tumor. However, some parts of the suprasellar craniopharyngioma were adherent to the walls of the third ventricle, which prevented us from achieving complete removal of the tumor without jeopardizing the adjacent neurovascular structures and the hypothalamus. When subsequent imaging revealed evidence of progressive enlargement of both the solid suprasellar component as well as a large cystic component extending into the interpeduncular space with compression of the brainstem, it was felt that a more direct approach utilizing an endoscopic endonasal transclival exposure to remove this lesion would be best for this patient. The patient tolerated both procedures well and has no current evidence of recurrence 2 months postoperatively. He 
is undergoing focal proton beam radiotherapy to the solid parasellar component of the residual lesion.

\section{Conclusions}

The simultaneous occurrence of a craniopharyngioma and a separate dermoid cyst is unusual and provocative. Furthermore, this association might indeed attest to the previous hypothesis that these lesions represent a continuum in a spectrum of ectodermally derived epithelial-lined cystic lesions, rather than a mere coincidence.

\section{References}

1. Alomari AK, Kelley BJ, Damisah E, Marks A, Hui P, DiLuna M, et al: Craniopharyngioma arising in a Rathke's cleft cyst: case report. J Neurosurg Pediatr 15:250-254, 2015

2. Asa SL, Kovacs K, Horvath E, Losinski NE, Laszlo FA, Domokos I, et al: Human fetal adenohypophysis. Electron microscopic and ultrastructural immunocytochemical analysis. Neuroendocrinology 48:423-431, 1988

3. Buslei R, Nolde M, Hofmann B, Meissner S, Eyupoglu IY, Siebzehnrübl F, et al: Common mutations of beta-catenin in adamantinomatous craniopharyngiomas but not in other tumours originating from the sellar region. Acta Neuropathol 109:589-597, 2005

4. Caldarelli M, Massimi L, Kondageski C, Di Rocco C: Intracranial midline dermoid and epidermoid cysts in children. J Neurosurg 100 (5 Suppl Pediatrics):473-480, 2004

5. Cheng ML, Chang SD, Pang D, Adler JR: Intracranial nasal dermoid sinus cyst associated with colloid cyst of the third ventricle. Case report and new concepts. Pediatr Neurosurg 31:201-206, 1999

6. Cobbs CS, Pitts LH, Wilson CB: Epidermoid and dermoid cysts of the posterior fossa. Clin Neurosurg 44:511-528, 1997

7. Fahlbusch R, Honegger J, Paulus W, Huk W, Buchfelder M: Surgical treatment of craniopharyngiomas: experience with 168 patients. J Neurosurg 90:237-250, 1999

8. Fenstermaker RA, Ganz E, Roessmann U: Giant invasive intracerebral dermoid tumor with subependymoma-like reaction: case report. Neurosurgery 25:646-648, 1989

9. Fernandez-Miranda JC, Engh JA, Pathak SK, Madhok R, Boada FE, Schneider W, et al: High-definition fiber tracking guidance for intraparenchymal endoscopic port surgery. J Neurosurg 113:990-999, 2010

10. Gormley WB, Tomecek FJ, Qureshi N, Malik GM: Craniocerebral epidermoid and dermoid tumours: a review of 32 cases. Acta Neurochir (Wien) 128:115-121, 1994

11. Harrison MJ, Morgello S, Post KD: Epithelial cystic lesions of the sellar and parasellar region: a continuum of ectodermal derivatives? J Neurosurg 80:1018-1025, 1994

12. Ikeda H, Suzuki J, Sasano N, Niizuma H: The development and morphogenesis of the human pituitary gland. Anat Embryol (Berl) 178:327-336, 1988

13. Jallo GI, Kothbauer K, Silvera VM: Colloid cyst and epidermoid cyst. Pediatr Neurosurg 35:48-49, 2001

14. Kurwale N, Kumar R, Sharma MC, Sharma BS: Suprasellar dermoid cyst associated with colloid cyst of the third ventricle: Disordered embryogenesis or a mere coincidence? J Neurosci Rural Pract 4:345-347, 2013

15. Larkin SJ, Ansorge O: Pathology and pathogenesis of craniopharyngiomas. Pituitary 16:9-17, 2013

16. Laws ER, Kanter AS: Rathke cleft cysts. J Neurosurg 101:571-572, 2004

17. Martinez-Barbera JP, Buslei R: Adamantinomatous craniopharyngioma: pathology, molecular genetics and mouse models. J Pediatr Endocrinol Metab 28:7-17, 2015
18. Miller DC: Pathology of craniopharyngiomas: clinical import of pathological findings. Pediatr Neurosurg 21 (Suppl 1):11-17, 1994

19. Ostrom QT, Gittleman H, Farah P, Ondracek A, Chen Y, Wolinsky Y, et al: CBTRUS statistical report: Primary brain and central nervous system tumors diagnosed in the United States in 2006-2010. Neuro Oncol 15 (Suppl 2):ii1-ii56, 2013

20. Park JG, Babu R, Kranz PG, McLendon RE, Adamson $\mathrm{C}$ : Intraaxial dermoid cyst of the medulla. J Neurosurg 119:442-445, 2013

21. Pascual JM, Carrasco R, Prieto R, Gonzalez-Llanos F, Alvarez F, Roda JM: Craniopharyngioma classification. J Neurosurg 109:1180-1183, 2008

22. Pascual JM, González-Llanos F, Barrios L, Roda JM: Intraventricular craniopharyngiomas: topographical classification and surgical approach selection based on an extensive overview. Acta Neurochir (Wien) 146:785-802, 2004

23. Prabhu VC, Brown HG: The pathogenesis of craniopharyngiomas. Childs Nerv Syst 21:622-627, 2005

24. Sener RN, Mechl M, Prokes B, Valek VA: Dermoid tumor with involvement of the frontal lobes. Acta Radiol 45:209211, 2004

25. Shanklin WM: The incidence and distribution of cilia in the human pituitary with a description of microfollicular cysts derived from Rathke's cleft. Acta Anat (Basel) 11:361-382, 1951

26. Shanklin WM: On the presence of cysts in the human pituitary. Anat Rec 104:379-407, 1949

27. Steno J: Microsurgical topography of craniopharyngiomas. Acta Neurochir Suppl (Wien) 35:94-100, 1985

28. Sweet WH: Radical surgical treatment of craniopharyngioma. Clin Neurosurg 23:52-79, 1976

29. Uglietta JP, Boyko OB, Rippe DJ, Fuller GN, Schiff SJ, Heinz ER: Intracerebral extension of nasal dermoid cyst: CT appearance. J Comput Assist Tomogr 13:1061-1064, 1989

30. Van Gilder JC, Schwartz HG: Growth of dermoids from skin implants to the nervous system and surrounding spaces of the newborn rat. J Neurosurg 26:14-20, 1967

31. VanGilder JC, Inukai J: Growth characteristics of experimental intracerebrally transplanted oral epithelium. J Neurosurg 38:608-615, 1973

32. Zada G, Lin N, Ojerholm E, Ramkissoon S, Laws ER: Craniopharyngioma and other cystic epithelial lesions of the sellar region: a review of clinical, imaging, and histopathological relationships. Neurosurg Focus 28(4):E4, 2010

\section{Disclosures}

The authors report no conflict of interest concerning the materials or methods used in this study or the findings specified in this paper.

\section{Author Contributions}

Conception and design: Chiocca, Abou-Al-Shaar, Laws. Acquisition of data: Abou-Al-Shaar, Zaidi, Russell-Goldman, Folkerth. Analysis and interpretation of data: all authors. Drafting the article: Abou-Al-Shaar, Laws. Critically revising the article: all authors. Reviewed submitted version of manuscript: all authors. Approved the final version of the manuscript on behalf of all authors: Chiocca. Study supervision: Chiocca.

\section{Correspondence}

E. Antonio Chiocca, Department of Neurosurgery, Brigham and Women's Hospital, 75 Francis St., Boston, MA 02115. email: eachiocca@partners.org. 